\title{
A new class of functions for integrating weighting means and OWA operators
}

\author{
Bonifacio Llamazares \\ Departamento de Economía Aplicada, Instituto de Matemáticas (IMUVA), \\ Universidad de Valladolid, Avda. Valle de Esgueva 6, 47011 Valladolid, Spain. \\ boni@eco.uva.es
}

\begin{abstract}
In this paper we introduce the semi-uninorm based ordered weighted averaging (SUOWA) operators, a new class of aggregation functions that integrates weighted means and OWA operators. To do this we take into account that weighted means and OWA operators are particular cases of Choquet integrals. So, the capacities associated to SUOWA operators are defined by using the values of the capacities associated to these functions and idempotent semi-uninorms.
\end{abstract}

Keywords: Weighted means, OWA operators, SUOWA operators, Choquet integrals, Semi-uninorms.

\section{Introduction}

Weighted means and ordered weighted averaging (OWA) operators (Yager [16]) are functions widely used in the aggregation processes. Although both are defined through weighting vectors, their behavior is quite different: Weighted means allow to weight each information source in relation to their reliability while OWA operators allow to weight the values according to their ordering.

Some authors, such as Torra [13] and Torra and Narukawa [15], have reported the need for both weightings. For instance, suppose we have several sensors to measure a physical property. On the one hand, sensors may be of different quality and precision, so a weighted mean type aggregation is necessary. On the other hand, to prevent a faulty sensor alters the measurement, we might take a OWA type aggregation where the maximum and minimum values are not considered.

Different aggregation functions have appeared in the literature to deal with this kind of situations. Special attention deserve the weighted OWA (WOWA) operators, introduced by Torra [13]. WOWA operators integrate weighted means and OWA operators in the sense that one of these functions is obtained when the other one has a "neutral" behavior, that is, its weighting vector is that of the arithmetic mean (the behavior of WOWA operators and other similar functions has been analyzed by Llamazares [10]).

The aim of this work is to introduce a new class of aggregation functions, the semi-uninorm based ordered weighted averaging (SUOWA) operators, that integrate the weighted means and the OWA operators in the above sense. To do 
this we take into account that weighted means and OWA operators are particular cases of Choquet integrals. So, the capacities associated to SUOWA operators are defined by using the values of the capacities associated to these functions and idempotent semi-uninorms.

The paper is organized as follows. In Section 2 we recall some basic properties of aggregation functions and the definitions of weighted means, OWA operators and Choquet integrals. Section 3 is devoted to the construction of capacities by means of which we can integrate weighted means and OWA operators in a new class of operators, the SUOWA operators. Finally, some concluding remarks are provided in Section 4.

\section{Preliminaries}

Throughout the paper we will use the following notation: $N=\{1, \ldots, n\}$; given $A \subseteq N,|A|$ will denote the cardinal of $A$; vectors will be denoted in bold; $\boldsymbol{\eta}$ will denote the vector $(1 / n, \ldots, 1 / n) ; \boldsymbol{x} \geq \boldsymbol{y}$ will mean $x_{i} \geq y_{i}$ for all $i \in N$; given $\boldsymbol{x} \in \mathbb{R}^{n}, x_{[1]} \geq \cdots \geq x_{[n]}$ and $x_{(1)} \leq \cdots \leq x_{(n)}$ will denote the components of $\boldsymbol{x}$ in decreasing and increasing order, respectively.

In the following definition we present some well-known properties usually demanded to the functions used in the aggregation processes.

Definition 1. Let $F: \mathbb{R}^{n} \longrightarrow \mathbb{R}$ be a function.

1. $F$ is symmetric if $F\left(x_{\sigma(1)}, \ldots, x_{\sigma(n)}\right)=F\left(x_{1}, \ldots, x_{n}\right)$ for all $\boldsymbol{x} \in \mathbb{R}^{n}$ and for all permutation $\sigma$ of $N$.

2. $F$ is monotonic if $\boldsymbol{x} \geq \boldsymbol{y}$ implies $F(\boldsymbol{x}) \geq F(\boldsymbol{y})$ for all $\boldsymbol{x}, \boldsymbol{y} \in \mathbb{R}^{n}$.

3. $F$ is idempotent if $F(x, \ldots, x)=x$ for all $x \in \mathbb{R}$.

4. $F$ is compensative (or internal) if $\min (\boldsymbol{x}) \leq F(\boldsymbol{x}) \leq \max (\boldsymbol{x})$ for all $\boldsymbol{x} \in \mathbb{R}^{n}$.

5. $F$ is homogeneous of degree 1 (or ratio scale invariant) if $F(\lambda \boldsymbol{x})=\lambda F(\boldsymbol{x})$ for all $\boldsymbol{x} \in \mathbb{R}^{n}$ and for all $\lambda>0$.

\subsection{Weighted means and OWA operators}

Weighted means and OWA operators are defined by vectors with non-negative components whose sum is 1 .

Definition 2. A vector $\boldsymbol{q} \in \mathbb{R}^{n}$ is a weighting vector if $\boldsymbol{q} \in[0,1]^{n}$ and $\sum_{i=1}^{n} q_{i}=1$.

Definition 3. Let $\boldsymbol{p}$ be a weighting vector. The weighted mean associated to $\boldsymbol{p}$ is the function $M_{\boldsymbol{p}}: \mathbb{R}^{n} \longrightarrow \mathbb{R}$ given by

$$
M_{\boldsymbol{p}}(\boldsymbol{x})=\sum_{i=1}^{n} p_{i} x_{i}
$$

Weighted means are continuous, monotonic, idempotent, compensative and homogeneous of degree 1 functions.

OWA operators were introduced by Yager [16] as a tool for aggregation procedures in multicriteria decision making. 
Definition 4. Let $\boldsymbol{w}$ be a weighting vector. The OWA operator associated to $\boldsymbol{w}$ is the function $O_{\boldsymbol{w}}: \mathbb{R}^{n} \longrightarrow \mathbb{R}$ given by

$$
O_{\boldsymbol{w}}(\boldsymbol{x})=\sum_{i=1}^{n} w_{i} x_{[i]} .
$$

OWA operators are continuous, symmetric, monotonic, idempotent, compensative and homogeneous of degree 1 functions.

\subsection{Choquet integrals}

Choquet integrals (see Choquet [2] and Murofushi and Sugeno [11]) are based on the notion of capacity. A capacity is similar to a probability measure but by replacing additivity by monotonicity (see also fuzzy measures in Sugeno [12]). Games are obtained when we drop the monotonicity property.

\section{Definition 5.}

1. A game $v$ on $N$ is a set function, $v: 2^{N} \longrightarrow \mathbb{R}$ satisfying $v(\varnothing)=0$.

2. A capacity (or fuzzy measure) $\mu$ on $N$ is a game on $N$ satisfying $\mu(A) \leq \mu(B)$ whenever $A \subseteq B$. Therefore, $\mu: 2^{N} \longrightarrow[0, \infty)$. The capacity is said to be normalized if $\mu(N)=1$.

The Choquet integral can be defined in a general context (see Choquet [2] and Murofushi and Sugeno [11]). However, we only consider the Choquet integral in the framework that we are dealing with here (see Grabisch et al. [8, p. 181]).

Definition 6. Let $\mu$ be a capacity on $N$. The Choquet integral with respect to $\mu$ is the function $\mathcal{C}_{\mu}: \mathbb{R}^{n} \longrightarrow \mathbb{R}$ given by

$$
\mathcal{C}_{\mu}(\boldsymbol{x})=\sum_{i=1}^{n} \mu\left(B_{(i)}\right)\left(x_{(i)}-x_{(i-1)}\right),
$$

where $B_{(i)}=\{(i), \ldots,(n)\}$ and, by convention, $x_{(0)}=0$.

It is worth noting that we have defined the Choquet integral for all vectors of $\mathbb{R}^{n}$ instead of nonnegative vectors because we are actually considering the asymmetric Choquet integral with respect to $\mu$ (on this, see Grabisch et al. [8, p. 182]). In addition to this, note that the Choquet integral can be defined with respect to games instead of capacities (see again Grabisch et al. [8, p. 181]). In this case, the Choquet integral satisfies the following properties (Grabisch et al. [8, p. 193 and p. 196]):

Remark 1. If $v$ is a game on $N$ and $\mathcal{C}_{v}$ is the Choquet integral with respect to $v$, then

1. $\mathcal{C}_{v}$ is continuous.

2. $\mathcal{C}_{v}$ is homogeneous of degree 1 . 
3. $\mathcal{C}_{v}$ is monotonic if and only if $v$ is a capacity.

4. $\mathcal{C}_{v}$ is idempotent when $v(N)=1$.

5. $\mathcal{C}_{v}$ is compensative when $v$ is a normalized capacity.

For the sake of similarity with OWA operators, in the sequel we show an equivalent representation of Choquet integral by means of decreasing sequences of values (see Torra [14]). Given $\boldsymbol{x} \in \mathbb{R}^{n}$, we can consider [.] and (.) so that $[i]=(n+1-i)$ for all $i \in N$. In this case,

$$
\mathcal{C}_{\mu}(\boldsymbol{x})=\sum_{i=1}^{n} \mu\left(A_{[i]}\right)\left(x_{[i]}-x_{[i+1]}\right),
$$

where $A_{[i]}=\{[1], \ldots,[i]\}$ and, by convention, $x_{[n+1]}=0$.

From the previous expression, it is straightforward to check that the Choquet integral can be written as

$$
\mathcal{C}_{\mu}(\boldsymbol{x})=\sum_{i=1}^{n}\left(\mu\left(A_{[i]}\right)-\mu\left(A_{[i-1]}\right)\right) x_{[i]},
$$

with the convention $A_{[0]}=\varnothing$. From this formula we can easily see that weighted means and OWA operators are specific cases of Choquet integral (see also Fodor et al. [3] and Grabisch [6,7]).

Remark 2. Let $\mu$ be a capacity on $N$.

1. $\mathcal{C}_{\mu}$ is the weighted mean $M_{\boldsymbol{p}}$ if $\mu\left(A_{[i]}\right)-\mu\left(A_{[i-1]}\right)=p_{[i]}$ for all $i \in N$, or, equivalently, $\mu\left(A_{[i]}\right)=\sum_{j=1}^{i} p_{[j]}$ for all $i \in N$. Therefore $\mu(A)=\sum_{i \in A} p_{i}$ for all $A \subseteq N$.

2. $\mathcal{C}_{\mu}$ is the OWA operator $O_{\boldsymbol{w}}$ if $\mu\left(A_{[i]}\right)-\mu\left(A_{[i-1]}\right)=w_{i}$ for all $i \in N$, or, equivalently, $\mu\left(A_{[i]}\right)=\sum_{j=1}^{i} w_{j}$ for all $i \in N$. Therefore $\mu(A)=\sum_{i=1}^{|A|} w_{i}$ for all $A \subseteq N$.

\section{Integrating weighting means and OWA operators}

Our aim is to find new functions based on the Choquet integral, $F_{\boldsymbol{p}, \boldsymbol{w}}$, that integrate weighted means and OWA operators in the following sense: $F_{\boldsymbol{p}, \boldsymbol{\eta}}=M_{\boldsymbol{p}}$ and $F_{\boldsymbol{\eta}, \boldsymbol{w}}=O_{\boldsymbol{w}}$ (see WOWA operators in Torra [13]).

If we represent the function $F_{\boldsymbol{p}, \boldsymbol{w}}$ as

$$
F_{\boldsymbol{p}, \boldsymbol{w}}(\boldsymbol{x})=\sum_{i=1}^{n}\left(\mu_{\boldsymbol{p}, \boldsymbol{w}}\left(A_{[i]}\right)-\mu_{\boldsymbol{p}, \boldsymbol{w}}\left(A_{[i-1]}\right)\right) x_{[i]},
$$

with $A_{[0]}=\varnothing$, then, according to Remark $2, F_{\boldsymbol{p}, \boldsymbol{w}}$ integrates weighted means and OWA operators when the capacity $\mu_{\boldsymbol{p}, \boldsymbol{w}}$ satisfies

$$
\mu_{\boldsymbol{p}, \boldsymbol{\eta}}(A)=\sum_{i \in A} p_{i} \quad \text { and } \quad \mu_{\boldsymbol{\eta}, \boldsymbol{w}}(A)=\sum_{i=1}^{|A|} w_{i}
$$


for all $A \subseteq N$.

In the next subsection we show a procedure for constructing capacities satisfying the conditions given by (1).

\subsection{Constructing capacities by using semi-uninorms}

Given $A \subseteq N$, weighted means and OWA operators are generated through normalized capacities defined by the values $\sum_{i \in A} p_{i}$ and $\sum_{i=1}^{|A|} w_{i}$, respectively. Therefore, our first intention is to consider a game on $N$ given as a function of these values; that is,

$$
\nu_{\boldsymbol{p}, \boldsymbol{w}}^{f}(A)=f\left(\sum_{i \in A} p_{i}, \sum_{i=1}^{|A|} w_{i}\right) .
$$

However, conditions given by (1) implies that, if $|A|=j$, then

$$
\nu_{\boldsymbol{\eta}, \boldsymbol{w}}^{f}(A)=f\left(\frac{j}{n}, \sum_{i=1}^{|A|} w_{i}\right)=\sum_{i=1}^{|A|} w_{i} \quad \text { and } \quad \nu_{\boldsymbol{p}, \boldsymbol{\eta}}^{f}(A)=f\left(\sum_{i \in A} p_{i}, \frac{j}{n}\right)=\sum_{i \in A} p_{i} ;
$$

that is, $j / n$ should be a neutral element of the function $f$. Since the neutral element of a function is unique, we should use different functions according to the cardinality of the set $A$. To avoid this, we make a transformation of the values $\sum_{i \in A} p_{i}$ and $\sum_{i=1}^{|A|} w_{i}$ taking into account the cardinality of the set $A$. So, when the set $A$ is non-empty, we consider the set function

$$
v_{\boldsymbol{p}, \boldsymbol{w}}^{f}(A)=|A| \cdot f\left(\frac{\sum_{i \in A} p_{i}}{|A|}, \frac{\sum_{i=1}^{|A|} w_{i}}{|A|}\right) .
$$

In this way, conditions given by (1) are satisfied when $f$ is a function with neutral element $1 / n$. When we look for functions with neutral elements, uninorms, introduced by Yager and Rybalov [17], appear in a natural way (see also Fodor et al. [5], and Fodor and De Baets [4]).

Definition 7. A function $U:[0,1]^{2} \longrightarrow[0,1]$ is a uninorm if it is symmetric, associative $(U(x, U(y, z))=U(U(x, y), z)$ for all $x, y, z \in[0,1])$, monotonic and possesses a neutral element $e \in[0,1](U(x, e)=x$ for all $x \in[0,1])$.

Nevertheless, for our purposes we can dispense with the symmetry and associativity properties. In this case we obtain semi-uninorms functions, introduced by Liu [9].

Definition 8. A function $U:[0,1]^{2} \longrightarrow[0,1]$ is a semi-uninorm if it is monotonic and possesses a neutral element $e \in[0,1](U(e, x)=U(x, e)=x$ for all $x \in[0,1])$. 
The set of semi-uninorms with neutral element $e \in[0,1]$ will be denoted by $\mathcal{U}^{e}$. Notice that semi-uninorms satisfy the following boundary conditions: $U(0,0)=0$ and $U(1,1)=1$.

Taking into account the above considerations, we can now define the game associated to two weighting vectors and a semi-uninorm.

Definition 9. Let $\boldsymbol{p}$ and $\boldsymbol{w}$ be two weighting vectors and let $U \in \mathcal{U}^{1 / n}$. The game associated to $\boldsymbol{p}, \boldsymbol{w}$ and $U$ is the set function $v_{\boldsymbol{p}, \boldsymbol{w}}^{U}: 2^{N} \longrightarrow \mathbb{R}$ defined by

$$
v_{\boldsymbol{p}, \boldsymbol{w}}^{U}(A)=|A| \cdot U\left(\frac{\sum_{i \in A} p_{i}}{|A|}, \frac{\sum_{i=1}^{|A|} w_{i}}{|A|}\right)
$$

if $A \neq \varnothing$, and $v_{\boldsymbol{p}, \boldsymbol{w}}^{U}(\varnothing)=0$.

It is easy to check that $v_{\boldsymbol{p}, \boldsymbol{w}}^{U}$ satisfies the conditions given by (1) and that $v_{\boldsymbol{p}, \boldsymbol{w}}^{U}(N)=1$. However, the game $v_{\boldsymbol{p}, \boldsymbol{w}}^{U}$ may not be a capacity; that is, it may not be monotonic as we show in the following example.

Example 1. Let $\boldsymbol{p}=(0.5,0.2,0.1,0.1,0.1)$ and $\boldsymbol{w}=(0.6,0.2,0,0,0.2)$. Given $U \in \mathcal{U}^{0.2}$, we have

- If $A=\{2\}$, then $v_{\boldsymbol{p}, \boldsymbol{w}}^{U}(A)=U(0.2,0.6)=0.6$.

- If $B=\{2,3,4,5\}$, then $v_{\boldsymbol{p}, \boldsymbol{w}}^{U}(B)=4 U(0.5 / 4,0.2)=0.5$.

Therefore, $A \subseteq B$ but $v_{\boldsymbol{p}, \boldsymbol{w}}^{U}(A)>v_{\boldsymbol{p}, \boldsymbol{w}}^{U}(B)$; that is, $v_{\boldsymbol{p}, \boldsymbol{w}}^{U}$ is not monotonic.

Nevertheless, it is relatively easy to obtain a capacity $\hat{v}$ from a game $v$. To do this, for each subset $A$ of $N$ we consider the maximum value of the set function over the subsets contained in $A$.

Definition 10. Let $v$ be a game on $N$. The capacity associated to $v$ is the set function $\hat{v}: 2^{N} \longrightarrow[0, \infty)$ given by

$$
\hat{v}(A)=\max _{B \subseteq A} v(B) .
$$

Some basic properties of $\hat{v}$ are given in the sequel.

Remark 3. Let $v$ be a game on $N$. Then:

1. If $v$ is a capacity, then $\hat{v}=v$.

2. If $v(A) \leq 1$ for all $A \subseteq N$ and $v(N)=1$, then $\hat{v}$ is a normalized capacity.

In addition to the previous properties, it is worth noting that if a game on $N$ fulfils the conditions given by (1), then the capacity associated to the game also satisfies these conditions.

Proposition 1. Let $\boldsymbol{p}$ and $\boldsymbol{w}$ be two weighting vectors and let $v_{\boldsymbol{p}, \boldsymbol{w}}$ be a game on $N$ such that $v_{\boldsymbol{p}, \boldsymbol{\eta}}(A)=\sum_{i \in A} p_{i}$ and $v_{\boldsymbol{\eta}, \boldsymbol{w}}(A)=\sum_{i=1}^{|A|} w_{i}$ for all $A \subseteq N$. Then, $\hat{v}_{\boldsymbol{p}, \boldsymbol{\eta}}(A)=\sum_{i \in A} p_{i}$ and $\hat{v}_{\boldsymbol{\eta}, \boldsymbol{w}}(A)=\sum_{i=1}^{|A|} w_{i}$ for all $A \subseteq N$. 
Proof. Given $A \subseteq N$,

$$
\begin{aligned}
& \hat{v}_{\boldsymbol{p}, \boldsymbol{\eta}}(A)=\max _{B \subseteq A} v_{\boldsymbol{p}, \boldsymbol{\eta}}(B)=\max _{B \subseteq A} \sum_{i \in B} p_{i}=\sum_{i \in A} p_{i}, \\
& \hat{v}_{\boldsymbol{\eta}, \boldsymbol{w}}(A)=\max _{B \subseteq A} v_{\boldsymbol{\eta}, \boldsymbol{w}}(B)=\max _{B \subseteq A} \sum_{i=1}^{|B|} w_{i}=\sum_{i=1}^{|A|} w_{i} .
\end{aligned}
$$

In accordance with the previous remarks, instead of using the games $v_{\boldsymbol{p}, \boldsymbol{w}}^{U}$ we will use the capacities associated with them.

Definition 11. Let $\boldsymbol{p}$ and $\boldsymbol{w}$ be two weighting vectors, let $U \in \mathcal{U}^{1 / n}$, and let $v_{\boldsymbol{p}, \boldsymbol{w}}^{U}$ be the game associated to $\boldsymbol{p}, \boldsymbol{w}$ and $U$. The capacity $\hat{v}_{\boldsymbol{p}, \boldsymbol{w}}^{U}$ associated to the game $v_{\boldsymbol{p}, \boldsymbol{w}}^{U}$ will be called the capacity associated to $\boldsymbol{p}, \boldsymbol{w}$ and $U$.

Notice that, by definition, $v_{\boldsymbol{p}, \boldsymbol{w}}^{U}(A) \geq 0$ for all $A \subseteq N$. Therefore, when $|A|=1$ we have $\hat{v}_{\boldsymbol{p}, \boldsymbol{w}}^{U}(A)=v_{\boldsymbol{p}, \boldsymbol{w}}^{U}(A)$.

Once we know how to obtain capacities, our next goal is to get normalized capacities. According to 2) of Remark 3 and Proposition 1, in order to obtain a normalized capacity on $N$ satisfying the conditions given by (1) it is sufficient to find a game $v_{\boldsymbol{p}, \boldsymbol{w}}$ on $N$ satisfying these conditions and such that $v_{\boldsymbol{p}, \boldsymbol{w}}(A) \leq 1$ for all $A \subseteq N$ and $v_{\boldsymbol{p}, \boldsymbol{w}}(N)=1$. However, the game $v_{\boldsymbol{p}, \boldsymbol{w}}^{U}$ may not satisfy the condition $v_{\boldsymbol{p}, \boldsymbol{w}}^{U}(A) \leq 1$ for all $A \subseteq N$, as we show in the following example.

Example 2. Let $\boldsymbol{p}=(0.5,0.2,0.1,0.1,0.1)$ and $\boldsymbol{w}=(0.6,0.2,0,0,0.2)$. Consider the semi-uninorm (see Calvo et al. [1, p. 11]) given by

$$
U(x, y)=\max (0, \min (1, x+y-0.2)) .
$$

It is easy to check that $U \in \mathcal{U}^{0.2}$. If $A=\{1,2\}$ we have

$$
v_{\boldsymbol{p}, \boldsymbol{w}}^{U}(A)=2 U(0.35,0.4)=2 \cdot 0.55=1.1>1 .
$$

Nevertheless, as we show in the following proposition, idempotent semiuninorms allow us to guarantee the condition $v_{p, w}^{U}(A) \leq 1$ for all weighting vectors $\boldsymbol{p}$ and $\boldsymbol{w}$ and for all $A \subseteq N$.

Proposition 2. Let $U \in \mathcal{U}^{1 / n}$. If $U$ is idempotent, then $v_{\boldsymbol{p}, \boldsymbol{w}}^{U}(A) \leq 1$ for all weighting vectors $\boldsymbol{p}$ and $\boldsymbol{w}$ and for all $A \subseteq N$.

Proof. Given $\boldsymbol{p}, \boldsymbol{w}$ two weighting vectors and a non-empty set $A$ of $N$, we have

$$
v_{\boldsymbol{p}, \boldsymbol{w}}^{U}(A)=|A| U\left(\frac{\sum_{i \in A} p_{i}}{|A|}, \frac{\sum_{i=1}^{|A|} w_{i}}{|A|}\right) \leq|A| U\left(\frac{1}{|A|}, \frac{1}{|A|}\right)=|A| \frac{1}{|A|}=1 .
$$

We will denote by $\mathcal{U}_{\mathrm{i}}^{e}$ the set of idempotent semi-uninorms with neutral element $e \in[0,1]$. It is worth noting that this class of functions has been characterized by Liu [9]. 
Proposition 3. Let $U \in \mathcal{U}^{e} . U$ is idempotent if and only if

$$
U(x, y)= \begin{cases}\min (x, y) & \text { if }(x, y) \in[0, e]^{2}, \\ \max (x, y) & \text { if }(x, y) \in[e, 1]^{2} \backslash\{(e, e)\}, \\ P(x, y) & \text { otherwise, }\end{cases}
$$

where $P:[0, e) \times(e, 1] \cup(e, 1] \times[0, e) \longrightarrow[0,1]$ is monotonic and $\min (x, y) \leq$ $P(x, y) \leq \max (x, y)$ for all $(x, y) \in[0, e) \times(e, 1] \cup(e, 1] \times[0, e)$.

Obviously, the smallest and the greatest idempotent semi-uninorm are, respectively, the following uninorms (which were given by Yager and Rybalov [17]):

$$
\begin{aligned}
U_{\min }(x, y) & = \begin{cases}\max (x, y) & \text { if }(x, y) \in[1 / n, 1]^{2}, \\
\min (x, y) & \text { otherwise },\end{cases} \\
U_{\max }(x, y) & = \begin{cases}\min (x, y) & \text { if }(x, y) \in[0,1 / n]^{2}, \\
\max (x, y) & \text { otherwise. }\end{cases}
\end{aligned}
$$

In the next subsection we formally define the SUOWA operators.

\subsection{SUOWA operators}

We now introduce SUOWA operators as the Choquet integrals with respect to the capacities $\hat{v}_{\boldsymbol{p}, \boldsymbol{w}}^{U}$.

Definition 12. Let $\boldsymbol{p}$ and $\boldsymbol{w}$ be two weighting vectors and let $U \in \mathcal{U}_{i}^{1 / n}$. The semi-uninorm based ordered weighted averaging (SUOWA) operator associated to $\boldsymbol{p}, \boldsymbol{w}$ and $U$ is the function $S_{p, \boldsymbol{w}}^{U}: \mathbb{R}^{n} \longrightarrow \mathbb{R}$ given by

$$
S_{\boldsymbol{p}, \boldsymbol{w}}^{U}(\boldsymbol{x})=\sum_{i=1}^{n} s_{i} x_{[i]},
$$

where $s_{i}=\hat{v}_{\boldsymbol{p}, \boldsymbol{w}}^{U}\left(A_{[i]}\right)-\hat{v}_{\boldsymbol{p}, \boldsymbol{w}}^{U}\left(A_{[i-1]}\right)$, with $\hat{v}_{\boldsymbol{p}, \boldsymbol{w}}^{U}$ the capacity associated to $\boldsymbol{p}, \boldsymbol{w}$ and $U, A_{[i]}=\{[1], \ldots,[i]\}$ and, by convention, $A_{[0]}=\varnothing$.

According to Remak 1, and since $\hat{v}_{\boldsymbol{p}, \boldsymbol{w}}^{U}$ are normalized capacities, SUOWA operators are continuous, monotonic, idempotent, compensative and homogeneous of degree 1 functions. In the sequel we show an example to illustrate these operators (the weighting vectors $\boldsymbol{p}$ and $\boldsymbol{w}$ are taken from Torra [13]).

Example 3. Let us to consider the weighting vectors $\boldsymbol{p}=(0.4,0.1,0.2,0.3)$ and $\boldsymbol{w}=(0.125,0.375,0.375,0.125)$. Besides $U_{\min }$ and $U_{\max }$, we are also going to use $U_{\mathrm{am}}$, the idempotent semi-uninorm obtained by means of the arithmetic mean:

$$
U_{\mathrm{am}}(x, y)=\left\{\begin{array}{l}
\min (x, y) \text { if }(x, y) \in[0,0.25]^{2}, \\
\max (x, y) \text { if }(x, y) \in[0.25,1]^{2} \backslash\{(0.25,0.25)\}, \\
(x+y) / 2 \text { otherwise. }
\end{array}\right.
$$


In Table 1 we show the games and the capacities associated to these idempotent semi-uninorms. Note that all the values of the games coincide with those of the capacities; that is, the games $v_{\boldsymbol{p}, \boldsymbol{w}}^{U_{\min }}, v_{\boldsymbol{p}, \boldsymbol{w}}^{U_{\mathrm{am}}}$ and $v_{\boldsymbol{p}, \boldsymbol{w}}^{U_{\max }}$ are actually capacities.

Table 1. Games and capacities associated to $U_{\min }, U_{\mathrm{am}}$ and $U_{\max }$

\begin{tabular}{lllllll} 
& \multicolumn{2}{c}{$U_{\min }$} & \multicolumn{2}{c}{$U_{\mathrm{am}}$} & \multicolumn{2}{c}{$U_{\max }$} \\
\hline \multicolumn{1}{c}{ Set } & $v_{\boldsymbol{p}, \boldsymbol{w}}^{U_{\min }}$ & $\hat{v}_{\boldsymbol{p}, \boldsymbol{w}}^{U_{\min }}$ & $v_{\boldsymbol{p}, \boldsymbol{w}}^{U_{\mathrm{am}}}$ & $\hat{v}_{\boldsymbol{p}, \boldsymbol{w}}^{U_{\mathrm{m}}}$ & $v_{\boldsymbol{p}, \boldsymbol{w}}^{U_{\max }}$ & $\hat{v}_{\boldsymbol{p}, \boldsymbol{w}}^{U_{\max }}$ \\
\hline$\{1\}$ & 0.125 & 0.125 & 0.2625 & 0.2625 & 0.4 & 0.4 \\
$\{2\}$ & 0.1 & 0.1 & 0.1 & 0.1 & 0.1 & 0.1 \\
$\{3\}$ & 0.125 & 0.125 & 0.125 & 0.125 & 0.125 & 0.125 \\
$\{4\}$ & 0.125 & 0.125 & 0.2125 & 0.2125 & 0.3 & 0.3 \\
$\{1,2\}$ & 0.5 & 0.5 & 0.5 & 0.5 & 0.5 & 0.5 \\
$\{1,3\}$ & 0.6 & 0.6 & 0.6 & 0.6 & 0.6 & 0.6 \\
$\{1,4\}$ & 0.7 & 0.7 & 0.7 & 0.7 & 0.7 & 0.7 \\
$\{2,3\}$ & 0.3 & 0.3 & 0.3 & 0.3 & 0.3 & 0.3 \\
$\{2,4\}$ & 0.4 & 0.4 & 0.4 & 0.4 & 0.4 & 0.4 \\
$\{3,4\}$ & 0.5 & 0.5 & 0.5 & 0.5 & 0.5 & 0.5 \\
$\{1,2,3\}$ & 0.7 & 0.7 & 0.7875 & 0.7875 & 0.875 & 0.875 \\
$\{1,2,4\}$ & 0.875 & 0.875 & 0.875 & 0.875 & 0.875 & 0.875 \\
$\{1,3,4\}$ & 0.9 & 0.9 & 0.9 & 0.9 & 0.9 & 0.9 \\
$\{2,3,4\}$ & 0.6 & 0.6 & 0.7375 & 0.7375 & 0.875 & 0.875 \\
$N$ & 1 & 1 & 1 & 1 & 1 & 1 \\
\hline
\end{tabular}

When $\boldsymbol{x}=(7,6,4,3)$, the values returned by the SUOWA operators by using the capacities $v_{\boldsymbol{p}, \boldsymbol{w}}^{U_{\min }}, v_{\boldsymbol{p}, \boldsymbol{w}}^{U_{\mathrm{am}}}$ and $v_{\boldsymbol{p}, \boldsymbol{w}}^{U_{\max }}$ are

$$
\begin{aligned}
S_{\boldsymbol{p}, \boldsymbol{w}}^{U_{\min }}(7,6,4,3) & =0.125 \cdot 7+0.375 \cdot 6+0.2 \cdot 4+0.3 \cdot 3=4.825, \\
S_{\boldsymbol{p}, \boldsymbol{w}}^{U_{\mathrm{am}}}(7,6,4,3) & =0.2625 \cdot 7+0.2375 \cdot 6+0.2875 \cdot 4+0.2125 \cdot 3=5.05, \\
S_{\boldsymbol{p}, \boldsymbol{w}}^{U_{\max }}(7,6,4,3) & =0.4 \cdot 7+0.1 \cdot 6+0.375 \cdot 4+0.125 \cdot 3=5.275 .
\end{aligned}
$$

However, when $\boldsymbol{x}=(6,7,3,4)$, the three functions take the same value:

$$
\begin{aligned}
S_{\boldsymbol{p}, \boldsymbol{w}}^{U_{\min }}(6,7,3,4) & =S_{\boldsymbol{p}, \boldsymbol{w}}^{U_{\mathrm{am}}}(6,7,3,4)=S_{\boldsymbol{p}, \boldsymbol{w}}^{U_{\max }}(6,7,3,4) \\
& =0.1 \cdot 7+0.4 \cdot 6+0.375 \cdot 4+0.125 \cdot 3=4.975 .
\end{aligned}
$$

\section{Conclusion}

In some practical cases it is necessary to combine values by using both a weighting mean and a OWA type aggregation. Although there exist in the literature a 
large number of aggregation operators, WOWA operators are the only ones that possess desirable properties for aggregation and allow us to deal with this kind of situations. As WOWA operators, the functions introduced in this paper are obtained from Choquet integrals with respect to normalized capacities. Therefore, SUOWA operators are continuous, monotonic, idempotent, compensative and homogeneous of degree 1 functions, and, consequently, they constitute an alternative to WOWA operators to deal with this kind of aggregation problems.

Acknowledgments. This work is partially supported by the Spanish Ministry of Economy and Competitiveness (Project ECO2012-32178) and ERDF.

\section{References}

1. Calvo, T., Kolesárová, A., Komorníková, M., Mesiar, R.: Aggregation operators: properties, classes and construction methods. In: Calvo, T., Mayor, G., Mesiar, R. (eds.) Aggregation Operators, pp. 3-104. Physica-Verlag, Heidelberg (2002)

2. Choquet, G.: Theory of capacities. Ann. Inst. Fourier 5, 131-295 (1953)

3. Fodor, J., Marichal, J.L., Roubens, M.: Characterization of the ordered weighted averaging operators. IEEE Trans. Fuzzy Syst. 3(2), 236-240 (1995)

4. Fodor, J., De Baets, B.: Uninorm basics. In: Wang, P.P., Ruan, D., Kerre, E.E. (eds.) Fuzzy Logic: A Spectrum of Theoretical \& Practical Issues, Studies in Fuzziness and Soft Computing, vol. 215, pp. 49-64. Springer, Berlin (2007)

5. Fodor, J.C., Yager, R.R., Rybalov, A.: Structure of uninorms. Int. J. Uncertain. Fuzziness Knowl.-Based Syst. 5(4), 411-427 (1997)

6. Grabisch, M.: Fuzzy integral in multicriteria decision making. Fuzzy Sets Syst. 69(3), 279-298 (1995)

7. Grabisch, M.: On equivalence classes of fuzzy connectives - the case of fuzzy integrals. IEEE Trans. Fuzzy Syst. 3(1), 96-109 (1995)

8. Grabisch, M., Marichal, J., Mesiar, R., Pap, E.: Aggregation Functions. Cambridge University Press, New York (2009)

9. Liu, H.W.: Semi-uninorms and implications on a complete lattice. Fuzzy Sets Syst. 191, 72-82 (2012)

10. Llamazares, B.: An analysis of some functions that generalizes weighted means and OWA operators. Int. J. Intell. Syst. 28(4), 380-393 (2013)

11. Murofushi, T., Sugeno, M.: A theory of fuzzy measures. Representation, the Choquet integral and null sets. J. Math. Anal. Appl. 159(2), 532-549 (1991)

12. Sugeno, M.: Theory of Fuzzy Integrals and its Applications. Phd thesis, Tokyo Institute of Technology (1974)

13. Torra, V.: The weighted OWA operator. Int. J. Intell. Syst. 12(2), 153-166 (1997)

14. Torra, V.: On some relationships between the WOWA operator and the Choquet integral. In: Proceedings of the Seventh International Conference on Information Processing and Management of Uncertainty in Knowledge-Based Systems (IPMU'98). pp. 818-824. Paris (France) (July 1998)

15. Torra, V., Narukawa, Y.: Modeling Decisions: Information Fusion and Aggregation Operators. Springer, Berlin (2007)

16. Yager, R.R.: On ordered weighted averaging operators in multicriteria decision making. IEEE Trans. Syst., Man, Cybern. 18(1), 183-190 (1988)

17. Yager, R.R., Rybalov, A.: Uninorm aggregation operators. Fuzzy Sets Syst. 80(1), 111-120 (1996) 